\begin{tabular}{|l|l|l|l|}
\hline Phytocoenologia & $17(1)$ & $105-124$ & Stuttgart-Braunschweig, February 14, 1989 \\
\hline
\end{tabular}

\title{
Phänologie der binnenländischen Halophytengesellschaften Niedersachsens
}

\author{
von Christiane JANSSEN und DiETMAR BRANDES, Braunschweig
}

mit 15 Figuren

\begin{abstract}
The phenology of the halophilic and halotolerant plant-communities of the most important inland salt marshes in Northwest-Germany is shown by means of symphenological diagrams. The communities in question are dominated by one or two species, which results in a very distinctive period of flowering. The predominating colours of the flowers are pink, violet and white. Two detailed vegetation maps and vegetation profiles are given to document the spatial relationship of the studied communities.
\end{abstract}

\section{Einleitung}

Zwei der Binnensalzstellen im südöstlichen Niedersachsen, die Barnstorfer Salzwiesen und die Seckertrift bei Jerxheim, haben aufgrund des Vorkommens von Salicornia ramosissima (Queller) überregionale Bedeutung, da alle anderen natürlichen Queller-Populationen im Binnenland der Bundesrepublik Deutschland erloschen sind. Es liegt auch aus diesem Grund nahe, die Phänologie dieser Salzstellen eingehender zu bearbeiten.

Den Untersuchungen zur Phänologie des $S$ a licornietum strictae an der Nordseeküste von GILLI (1950) und der Arbeit über die Binnensalzstellen in Lothringen von DUVIGNEAUD (1967), die einige Anmerkungen zur Phänologie enthält, fehlen phänologische Diagramme völlig.

Umfassende phänologische Untersuchungen von Pflanzengesellschaften liegen bisher meist von artenreichen, blumenbunten Beständen vor, wobei phänologische Diagramme relativ selten veröffentlicht wurden. Als erste Darstellung vollständiger Diagramme einer terrestrischen Pflanzengemeinschaft gilt nach SCHENNIKOW (1932) die Arbeit von GAMS (1918).

Die Phänologie der Säume wurde von DierschKE (1974), die der Sandtrokkenrasen von JECKEL (1984) ausführlich dargestellt. FOLLEKRUG (1969) und KRATOCHWIL $(1983,1984)$ bearbeiteten die jahreszeitliche Staffelung der Blühaspekte von Glatthaferwiesen bzw. Halbtrockenrasen. Bergwiesengesellschaften wurden von VOGEL (1981). Streuwiesen von Weber \& PFadenhader (1987), Hochstaudenfluren von OTTE (1986) und einige Ruderalgesellschaften von GÓdDE (1986) bearbeitet. Phänologische Diagramme eines M eli co - F a ge t $\mathrm{u}$ m s finden sich bei FULLEKRUG (1967).

Methodische Fragen der Phänologie wurden ausführlich bei SCHENNIKow (1932) und DIERSCHKE (1972) diskutiert. 
Die Bibliographien phänologischer Diagramme von BALÁTOVÁ-TULÁČKovÁ (1970) und TOXEN \& WOJTERSKA (1977) enthalten weitere Literaturhinweise.

\section{Methode}

Für die phänologischen Diagramme wurden Vegetationsaufnahmen angefertigt, in denen die Artmächtigkeit getrennt nach generativer und vegetativer Deckung aufgeführt wurde. Zur Beschreibung des Entwicklungszustandes der Pflanze diente der phänologische Aufnahmeschlüssel von DIERSCHKE (1972), der getrennt für Kräuter und Gräser jeweils für den vegetativen und generativen Zustand eine 10-teilige Skala enthält.

Die phänologischen Aufnahmen wurden von Mai bis Oktober 1984 von solchen Beständen angefertigt, die die entsprechende Pflanzengesellschaft gut charakterisieren. Das Aufnahmematerial wurde anschließend zu symphänologischen Diagrammen (vgl. DIERSCHKE 1972) zusammengestellt. Dabei erfaßt ein vollständiges symphänologisches Diagramm alle Arten einer Gesellschaft, während ein unvollständiges symphänologisches Diagramm vor allem die für die Phänologie entscheidenden Arten berücksichtigt und solche mit geringem Deckungsgrad vernachlässigt.

Ein Überblick über die auf den Salzstellen vorkommenden Pflanzengesellschafschaften einschließlich pflanzensoziologischer Tabellen wurde bereits zu einem früheren Zeitpunkt gegeben (JANSSEN 1986).

\section{Phänologische Diagramme der Halophytenfluren und halotoleranter Kon- taktgesellschaften}

Die Phänologie der untersuchten Salzwiesen wird häufig durch die Dominanz von ein oder zwei Arten geprägt, da es sich in der Regel um recht artenarme Gesellschaften handelt. TUXEN (1961) weist bereits auf den fehlenden Blütenreichtum von Pflanzengesellschaften an Extremstandorten - wie etwa Salzstellen - - hin.

Legende zu Fig. 1-11:

Deckung

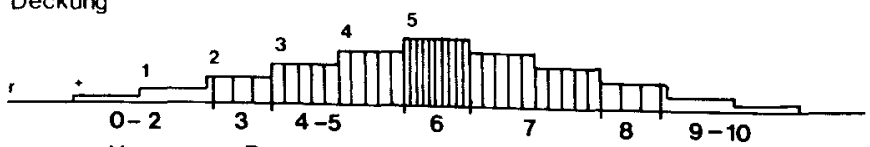

Vegetative Entwicklungsphase

Blütenfarbe
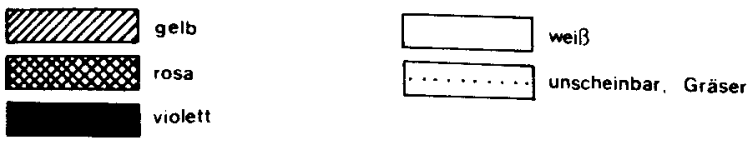


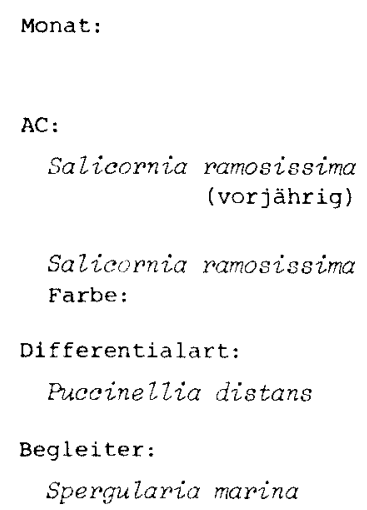

\section{Differentialart:}

Puccineliza distans

Begleiter:

Spergularia marina

Deckung (z):

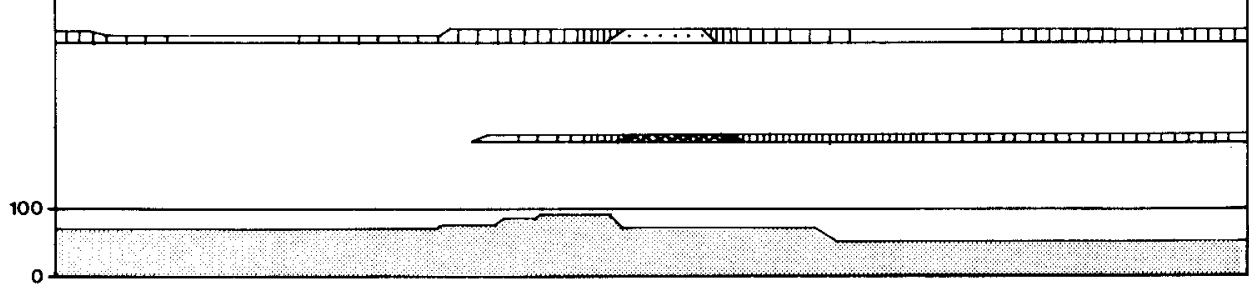


Monat:

AC:

Puccine Zlia distans $\mathrm{KC}$ :

Aster tripolium

Spergutaria marina

Glaux maritima

Begleiter:

Salicomia ramosissima (vorjährig)

Salicomia ramosissima

Atriplex hastata

Trip Zeurospermim inodorum

Apium graveotens

Poa trivialis

Agrostis stozonifera agg.

Deckung $(\%)$ :

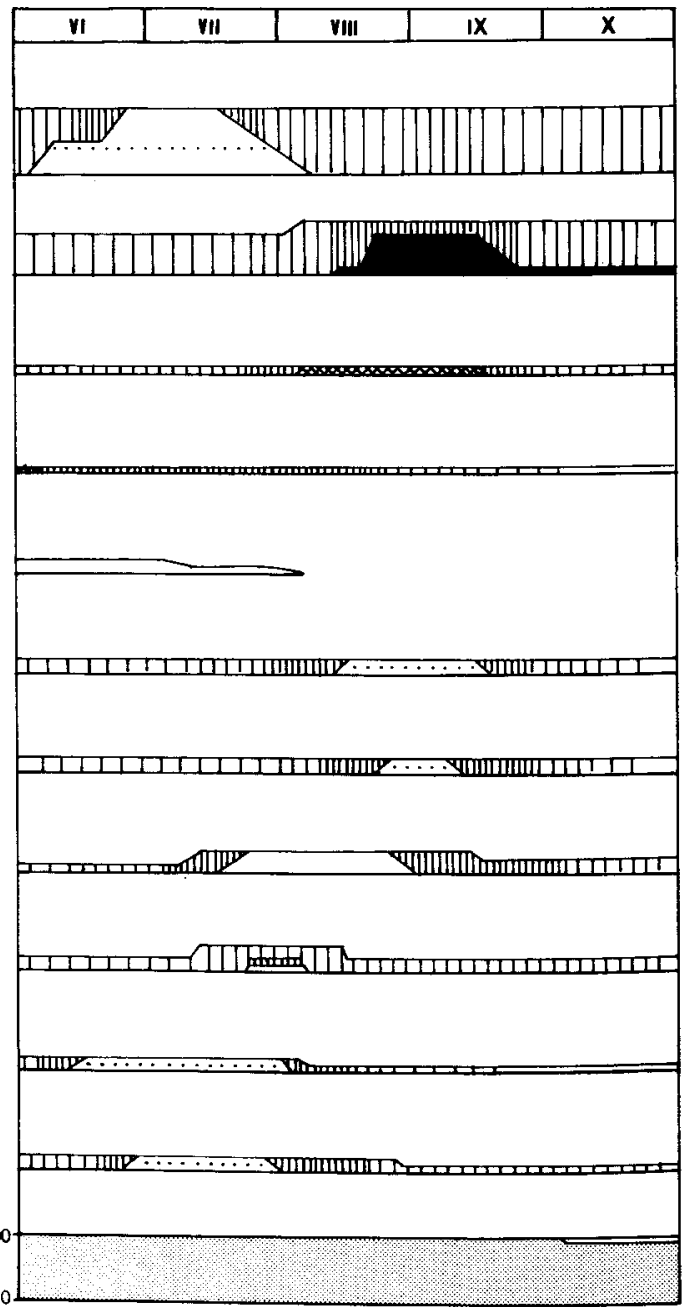

Fig. 2. Vollständiges symphänologisches Diagramm des Puccinellietum distantis I, Typische Variante, Jerxheim 1984.

Daraus ergibt sich auch, daß die jeweiligen Halophytenfluren meist nur eine einzige ausgeprägte Blühwelle aufweisen, die recht spät im Jahr liegen kann. Die auffälligsten Blühphasen der einzelnen Gesellschaften wechseln einander mehr oder minder ab, so daß praktisch von Mai bis Oktober vorherrschende Aspekte beobachtet werden können (vgl. JANSSEN 1986). 
Monat:

$\mathrm{AC}$ :

Puccinellia distans

Differentialart:

Aster tripolium

Asteretea-Art:

Sperguzaria marina

Begleiter:

Salicomia ramosissima

Tripleurospermum inodorum

Atriplex hastata

Apium graveotens

Lolium perenne

Polygonum avicuzare

Deckung (s):

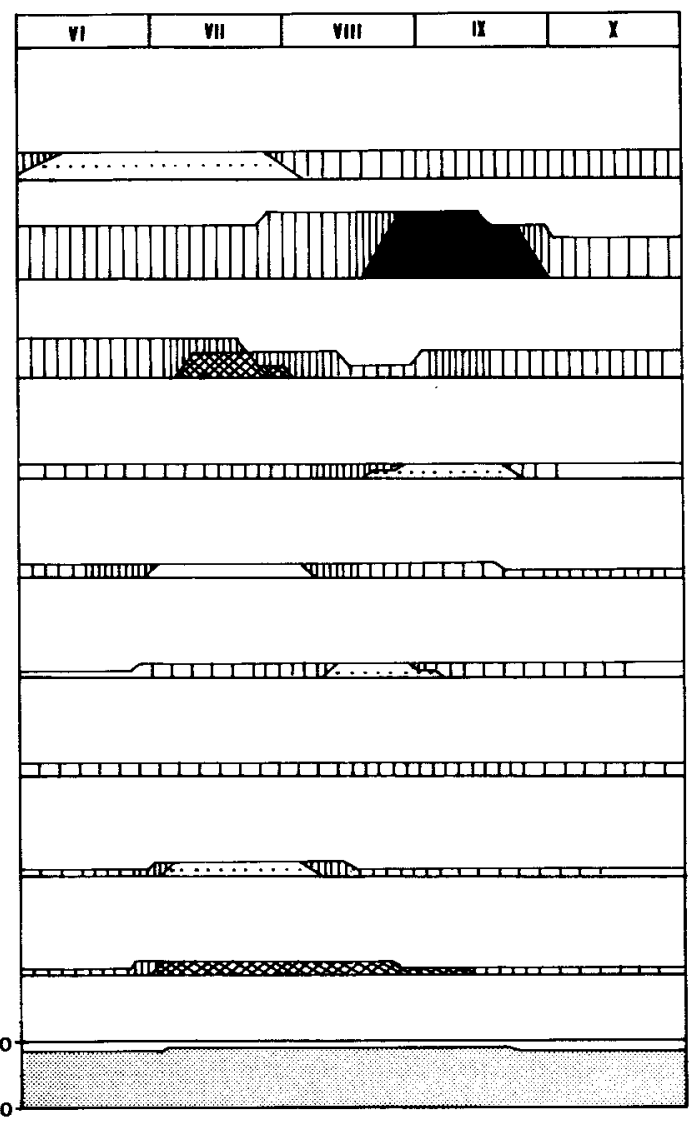

AuBerdem nichtblühend: $r$ Cirsium arvense, $r^{\circ}$ Lamium album.

Fig. 3. Vollständiges symphänologisches Diagramm des Puccinellietum distantis II, Variante von Aster tripolium, Jerxheim 1984.

Bereits im Mai blüht Glaux maritima, die aber aufgrund ihrer geringen Größe unauffällig ist und in mehreren Gesellschaften als Begleiter auftritt. Bis Ende Oktober fällt der weiße Fruchtpappus der Salz-Aster im P u c c in ell i e t u m $\mathrm{d}$ i s $t$ a $n$ t i s, Variante von Aster tripolium, auf. So üben die Salzwiesen trotz des geringen Arten reichtums einen geheimnisvollen ästhetischen Reiz aus.

In artenreichen Gesellschaften lösen sich oft mehrere Blühwellen ab, wie etwa DIERSCHKE (1974) fürdie T r i f o l i o-G e r a n i e t e a-Gesellschaften belegte. 

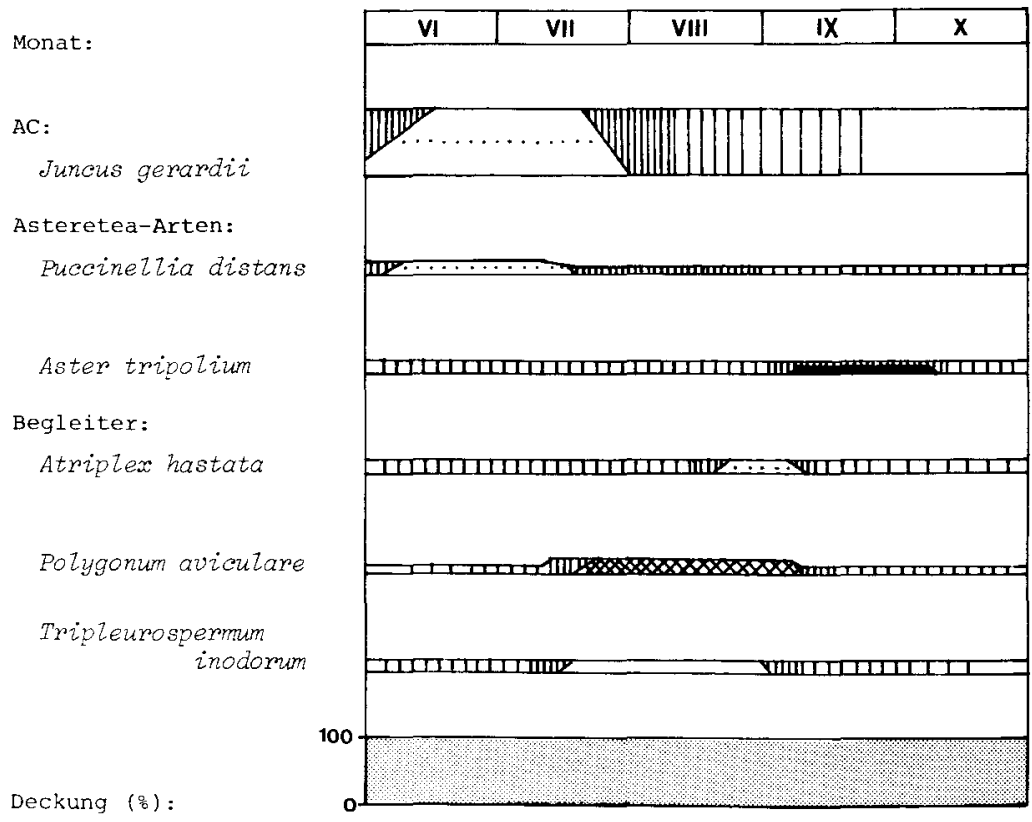

Außerdem nichtblühend: + Poa trivialis, + Agropyron repens,

+ Apium graveolens, r Sonchus spec., r Rumex cf. conglomeratus.

Fig. 4. Vollständiges symphänologisches Diagramm des Juncetum gerardii, Jerxheim 1984.

Auf den untersuchten Salzstellen sind die meisten der dominierenden Arten anemophil (Salicornia ramosissima, Puccinellia distans). Nur knapp $50 \%$ der Halophyten werden hier von Insekten bestäubt, allerdings treten viele dieser Sippen -- abgesehen von Aster tripolium - mit geringen Deckungsgraden auf.

SCHWABE \& KRATOCHWIL (1984) geben für Grüppenbeete auf Borkum $62 \%$ $(\mathrm{N}=18)$ anemophile und $38 \%(\mathrm{~N}=10)$ entomophile Salzrasen-Pflanzen an. Daß dic Anzahl der vom Wind bestäubten Sippen an der Küste sehr hoch ist, hängt sicher mit den dort ungünstigen Umweltbedingungen für Insekten (Gezeiten, ständiger Wind) zusammen.

Die vorherrschenden Blütenfarben der binnenländischen Salzrasen sind rosa, violett und weiß. An den untersuchten Stellen wurden als Aster tripolium- und Apium graveolens-Blüten besuchende Insekten vor allem Schwebfliegen in hoher Individuenzahl beobachtet. Nach SCHWABE \& KRATOCHWIL spielen an der Küste dagegen Hummeln eine vorherrschende Rolle. 
Monat:

Kriechrasenarten:

Agropyron repens

Apium graveolens

Rumex crispus

Begleiter:

Aster tripolizm

Tripteurospermum inodorum

Poa trivialis

Galium aparine

Cirsium arvense

Atriplex hastata

Deckung (o) :

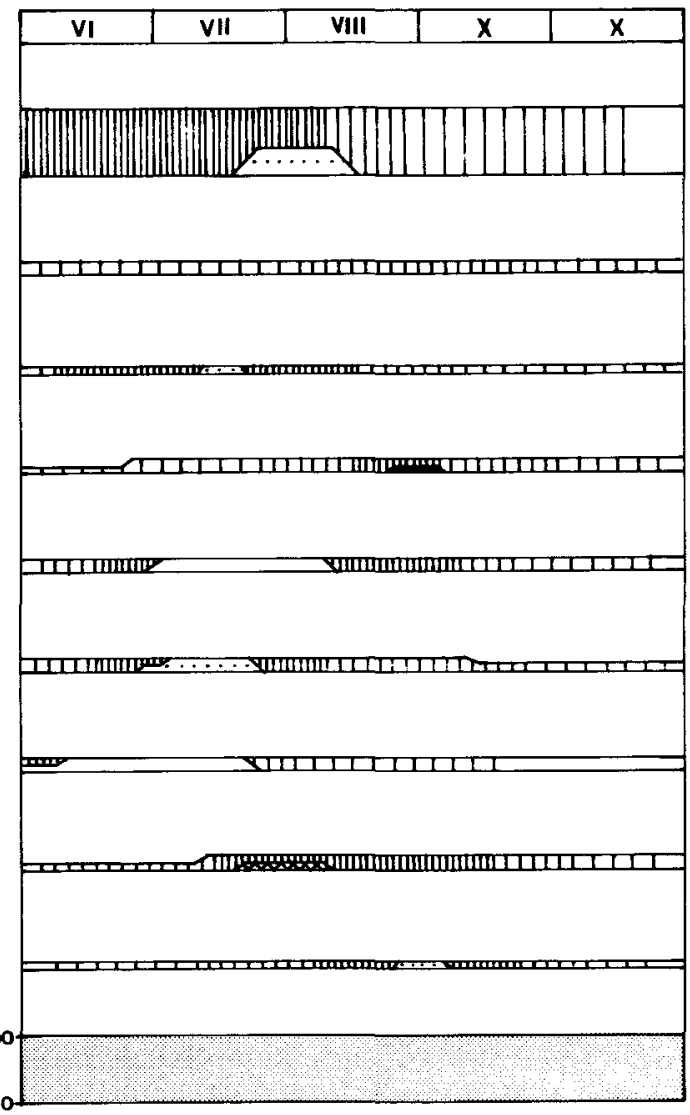

Fig. 5. Unvollständiges symphänologisches Diagramm der Agropryron repens-Gesellschaft, Jerxheim 1984.

\subsection{Halophytenfluren s. str.}

Das Puccinellio distantis-Salicornietum ramosissi$\mathrm{m}$ a e Tx. 1974, das von den vorkommenden Halophytengesellschaften die höchsten Salzkonzentrationen im Boden toleriert und meist kreisförmig um ein vegetationsfreies Zentrum angeordnet ist (vgl. Fig. 12), ist eine extrem artenarme Gesellschaft, die bis Mitte August recht unscheinbar wirkt. Erst dann beginnt allmählich die rote Herbstfärbung der aspektbildenden Art (Salicornia ramosissima), bis sich der Queller-Teppich im Oktober durch ein intensives Rot deutlich von der Umgebung abhebt (Fig. 1). Ende Oktober, spätestens nach dem ersten Frost geht das Rot der Queller-Pflanzen rasch in Braun über. Die abgestorbenen grauen Queller-Pflanzen des Vorjahres werden nur. sehr langsam zersetzt und bilden auf 


\section{Monat:}

Kennart:

Hordeum secalinum

Kriechrasen-

und Wiesenarten:

Agropyron repens

Carex otrubae

Poa trivialis

Festuca mibra

Agrostis

stozonifera agg.

Potentizla ansemina

Plantago major

Begleiter:

Giaix maritima

Triglochin maritimum

Encoineliza distans

Deckung ( $(\mathrm{g})$ :

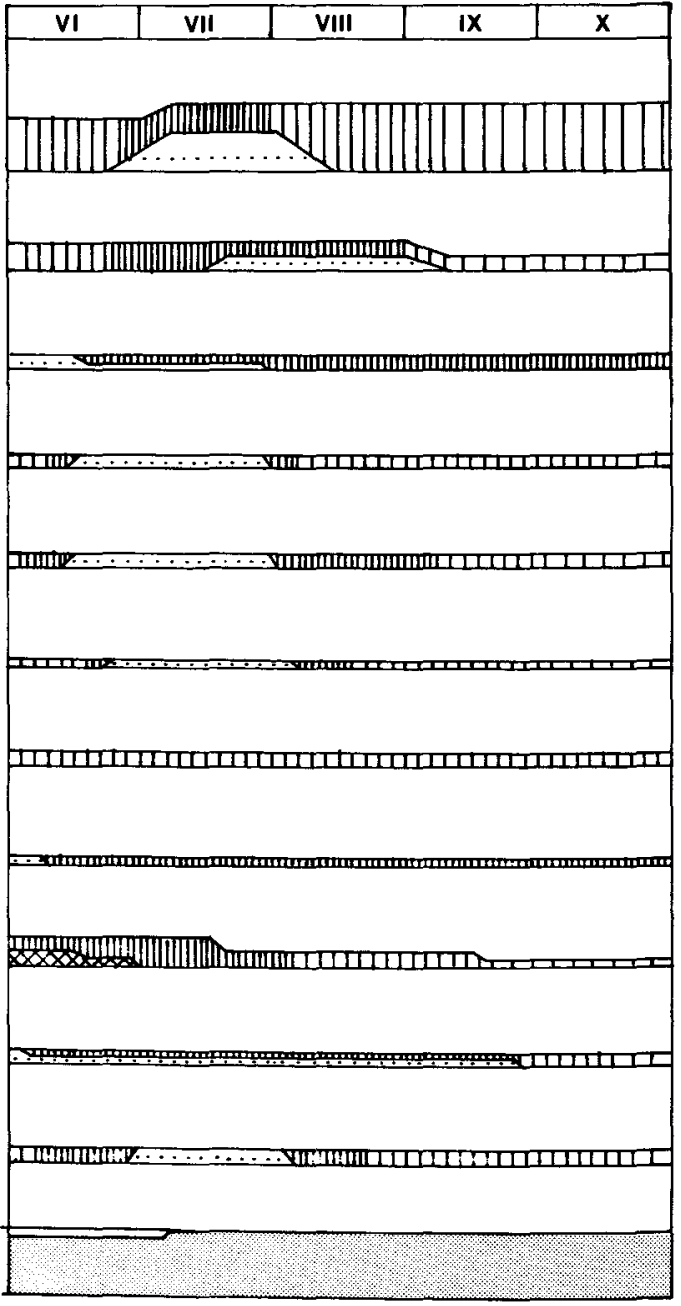

Außerdem nichtblühend: + Deschampsia cespitosa.

Fig. 6. Vollständiges symphänologisches Diagramm eines Hordeum secalinum-Bestandes, Barnstorf 1984. 


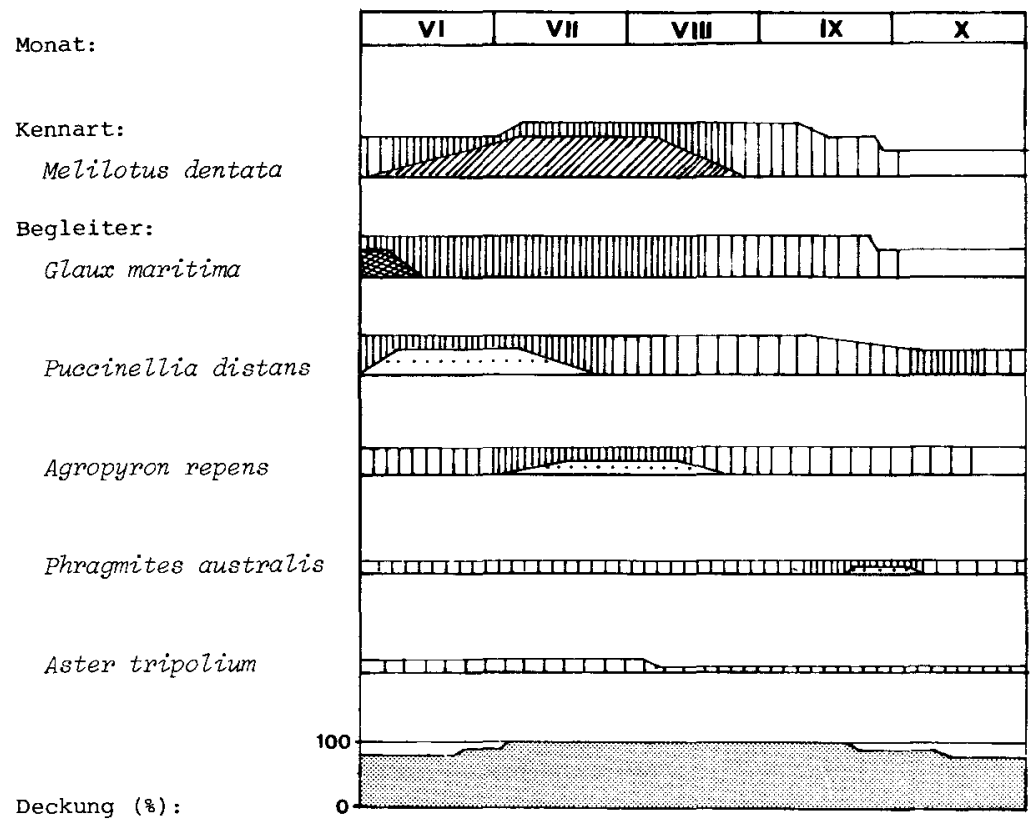

Fig. 7. Vollständiges symphänologisches Diagramm eines Melilotus dentata-Bestandes, Jerxheim 1984.

den nicht beweideten Flächen mindestens ein Jahr lang einen dünnen Filz. Auf den Wiesen in Barnstorf werden sie dagegen durch den Tritt des Weideviehs zerkleinert und sind ab Ende Juli verschwunden (Fig. 1). Das Diagramm zeigt die Subassoziation von Puccinellia distans, in der neben Salicornia ramosissima meist Puccinellia distans, Spergularia marina und Aster tripolium vorkommen.

Etwas artenreicher ist das sich direkt daran anschließende $\mathrm{P}$ u c c in elli e t u m distantis Feekes (1934) 1943. Die Typische Variante (Fig. 2) erreicht bereits Ende Juni ihren ersten Höhepunkt, wenn der dominierede Salzschwaden die grünlich-violetten Blüten schiebt. Hellviolette Blütenköpfe der Salz-Aster bestimmen anschließend das Bild. Abgesehen von Tripleurospermum inodorum tragen alle weiteren Arten kaum zum Erscheinungsbild dieser Gesellschaft bei.

Die $\mathrm{V}$ a $\mathrm{r}$ i a $\mathrm{n}$ t e von Aster tripolium (Fig. 3) zeigt demgegenüber nur eine Blühwelle von Mitte August bis Ende September, wenn die Fläche von den Blüten der Salz-Aster übersät ist. Zu dieser Zeit liegt über den Salzwiesen ein intensiver, leicht süßlicher Duft. Der weiße Pappus von Aster tripolium ist bis in den November recht auffällig. Nach der Blüte von Puccinellia distans beginnen die Halme und Blätter allmählich zu vergilben, allerdings treiben die Pflanzen im Oktober noch grüne oberirdische Ausläufer. 
Monat:

Centaurium pulchelzum

Plantago major ssp. winteri

Agropyron repens

Puecinellia distans

Lolium perenne

Potentizza anserina

Poa pratensis

Trip zeurospermu inodorum

Deckung (\%):

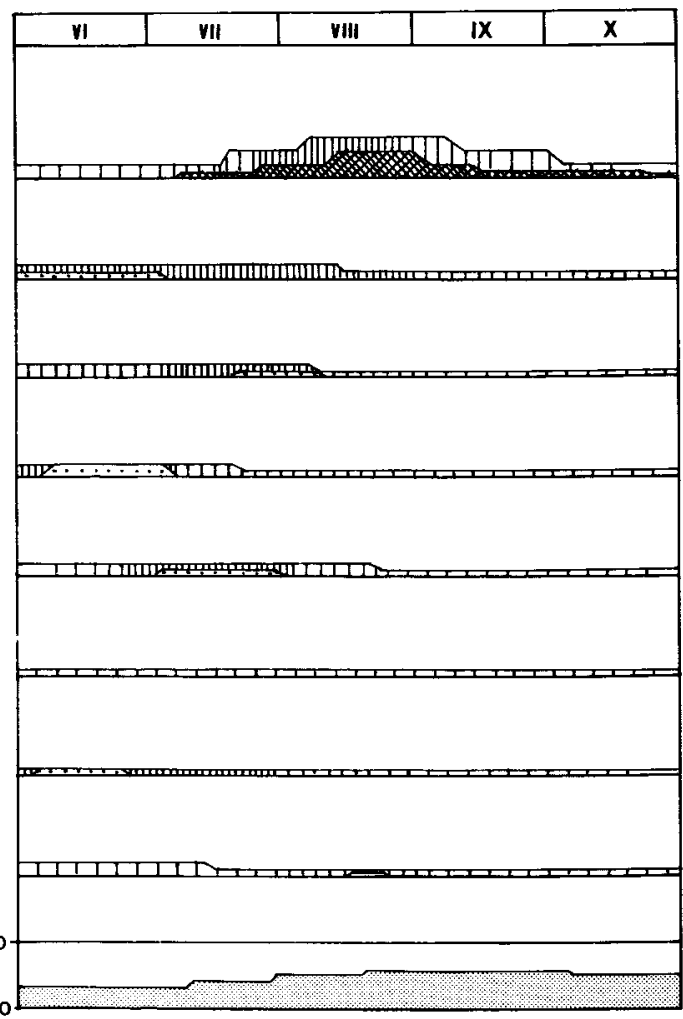

Fig. 8. Vollständiges symphänologisches Diagramm eines Centaurium pulcbellum-Bestandes, Jerxheim 1984.

Der Blühaspekt des J u n c e t u m g e r a r d i i Warming 1906, das inselartig in die Salzmieren-Rasen ( $\mathrm{P}$ u c c in e $1 \mathrm{l}$ i e $\mathrm{t} \mathrm{um} \mathrm{distantis)} \mathrm{eingestreut}$ ist, beginnt bereits Ende Mai und wird von den bräunlich-violetten Blüten der namengebenden Art bestimmt (Fig. 4). Nach der Blüte vergilben Halme und Blätter auffallend rasch, Ende September sind die Pflanzen oberirdisch abgestorben. Da Juncus gerardii-Horste einen dicken Wurzelfilz ausbilden und die abgestorbenen Halme dem Boden dicht aufliegen, kommen alle anderen Arten nur mit geringer Deckung und verminderter Vitalität vor, gelangen aber dennoch zur Blüte.

\subsection{Halotolerante Kontaktgesellschaften}

Zu den Kriechrasen ( $\mathrm{T}$ r if olio-Agrostietalia bzw. Agrostiet e a s t o lo n i f e r a e) zählen die Agropyron repens-Gesellschaft und Hor- 


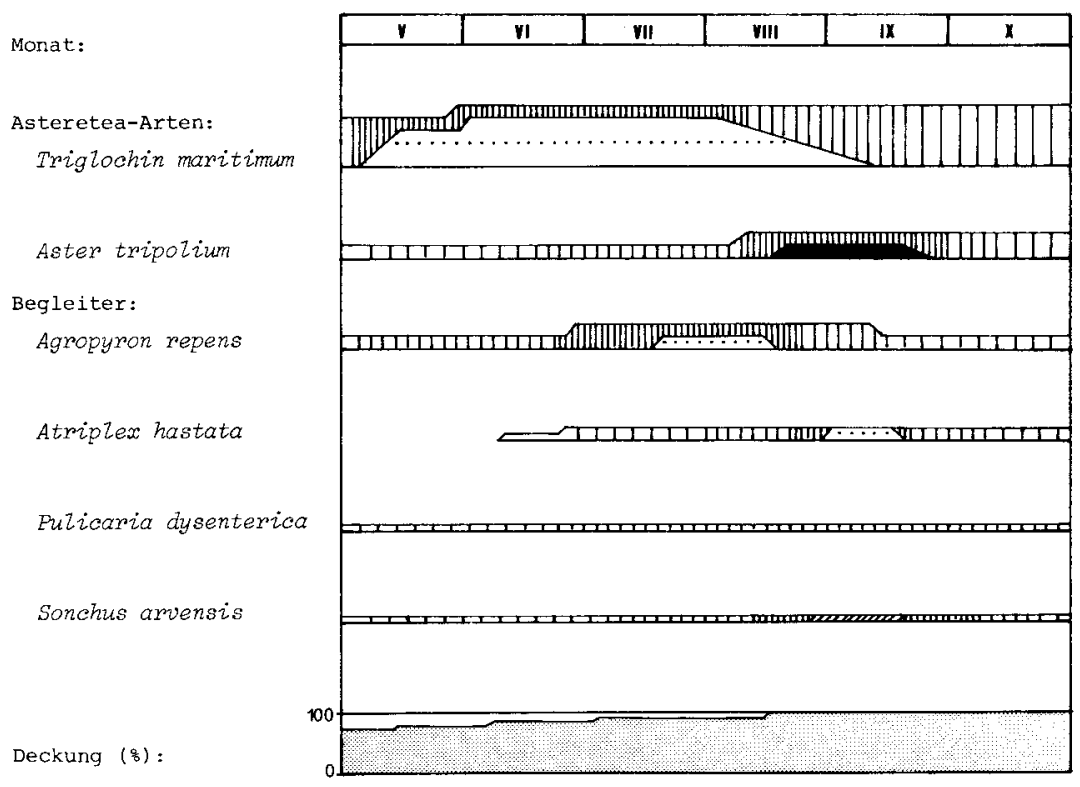

Fig. 9. Vollständiges symphänologisches Diagramm eines Triglocbin maritimum-Bestandes im Salzgraben östlich von Salzdahlum 1984.

deum secalinum-Bestände (Fig. 5, 6), in denen die Gräser aspektbildend auftreten und die Begleiter nur geringe Deckungsgrade erreichen.

Durch blaßgelbe Blüten treten Melilotus dentata-Herden (Fig. 7) von Anfang Juni bis Mitte August in Erscheinung.

Der Centaurium pulcbellum-Bestand (Fig. 8), der auf einem schwach salzbeeinflußten Trampelpfad gedeiht, steht ökologisch den Zwergbinsengesellschaften nahe, wenn auch hier alle Begleiter zu den ausdauernden Arten zählen. Die seltenen Störungen durch Tritt, der im Frühjahr feuchte und im Sommer austrocknende Boden und der geringe Salzgehalt gewährleisten, daß Centaurium pulchellum als Therophyt stets offene Stellen findet. Die Blüte erreicht zwar im August ihren Höhepunkt, doch ist die Blühperiode mit fast 4 Monaten erstaunlich lang. Die Begleiter treten wegen der ungünstigen Bedingungen mit deutlich verminderter Vitalität auf.

Salzbeeinflußte Gräben werden im Untersuchungsgebiet häufig von Triglochin maritimum-Dominanzbeständen (Fig. 9) oder dem B o l b o s ch o e n e t u m m a r i t i m i (Fig. 10) besiedelt. Die unscheinbare Blüte von Triglochin maritimum dauert von Mai bis Anfang September und wird von den Farbtupfen der Aster-Blüten abgelöst. Sowohl Triglocbin maritimum als auch Bolboschoenus maritimus verfügen über eine erfolgreiche vegetative Vermehrung, so daß sie an günstigen Standorten dichte Bestände bilden und andere Arten nur mit geringen Deckungsgraden zulassen. 


Monat:
AC:
Bolboschoenus maritimus
Begleiter:
Puccineliza distans
Atriplex hastata
Aster tripolium
Tripleurospermum
inodomm
Pulicaria dysenterica
Artemisia vuigaris
Postuntites mibra
Privialis

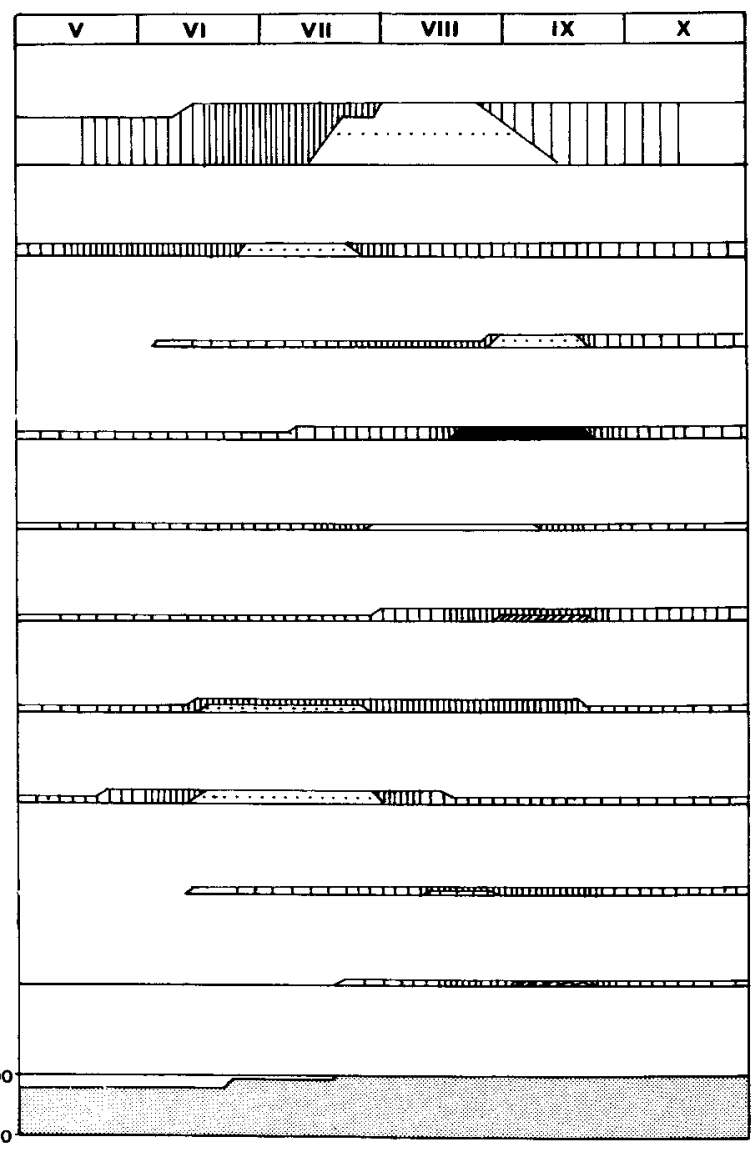

Fig. 10. Vollständiges symphänologisches Diagramm des Bolboschoenetum maritimi in einem Salzgraben östlich von Salzdahlum 1984.

Phragmites australis baut auf den schwächer salzhaltigen Böden bei fehlendem Beweidungsdruck Halophytenfluren regelrecht $a b$. Die Bestände, die viele halotolerante Begleiter aufweisen (Fig. 11), schieben sich als breites Band in die Seckertrift hinein (vgl. Fig. 14). Von vergleichbaren Beobachungen berichten SCHMEISKy (1977) und AlteHAGE \& ROSSMANN (1940).

\section{Vegetationskartierung}

Die Vegetationskarten (Fig. 12,14) und die Vegetationsprofile (Fig. 13, 15) vermitteln einen Eindruck vom räumlichen Nebeneinander der Halophytengesell- 


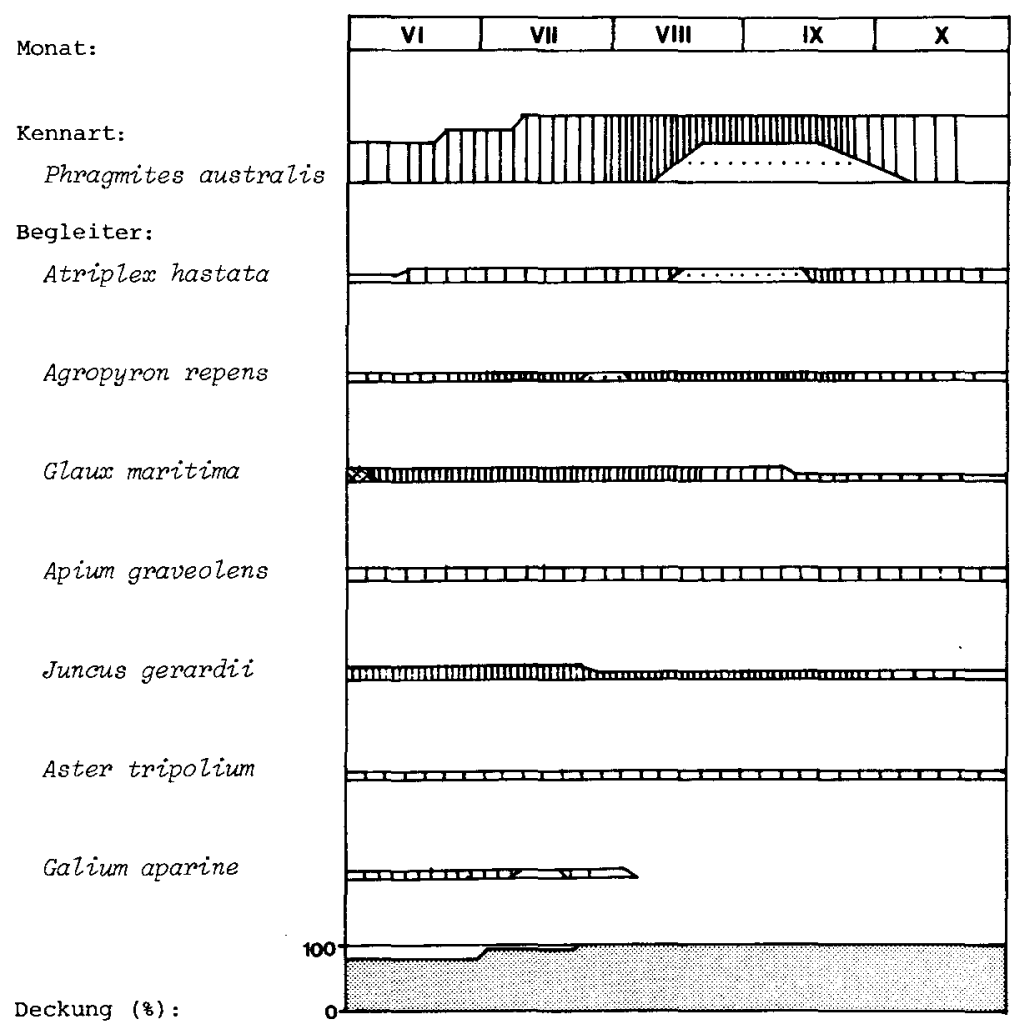

Fig. 11. Vollständiges symphänologisches Diagramm eines Pbragmites australis-Bestandes, Jerxheim 1984.

schaften s.l. Weiterhin dienen besonders die Vegetationskarten als wichtiges Vergleichsmaterial für spätere Untersuchungen. Derzeit wird u.a. die Veränderung der Artenzusammensetzung bei ausbleibender Beweidung näher untersucht.

Zusammenfassung. Die Phänologie der Halophytenfluren und halotoleranter Kontaktgesellschaften der bedeutendsten Binnensalzstellen Nordwestdeutschlands wird an $\mathrm{Hand}$ von symphänologischen Diagrammen vorgestellt und diskutiert. Die vorkommenden Gesellschaften zeichnen sich meist durch die Dominanz von ein oder zwei Arten aus und weisen dementsprechend eine ausgeprägte Blühwelle auf. Die vorherrschenden Blütenfarben sind rosa, violett und weiß. Zwei detaillierte Vegetationskarten und. Vegetationsprofile vermitteln einen Eindruck vom räumlichen Nebeneinander der untersuchten Gesellsch aften. 


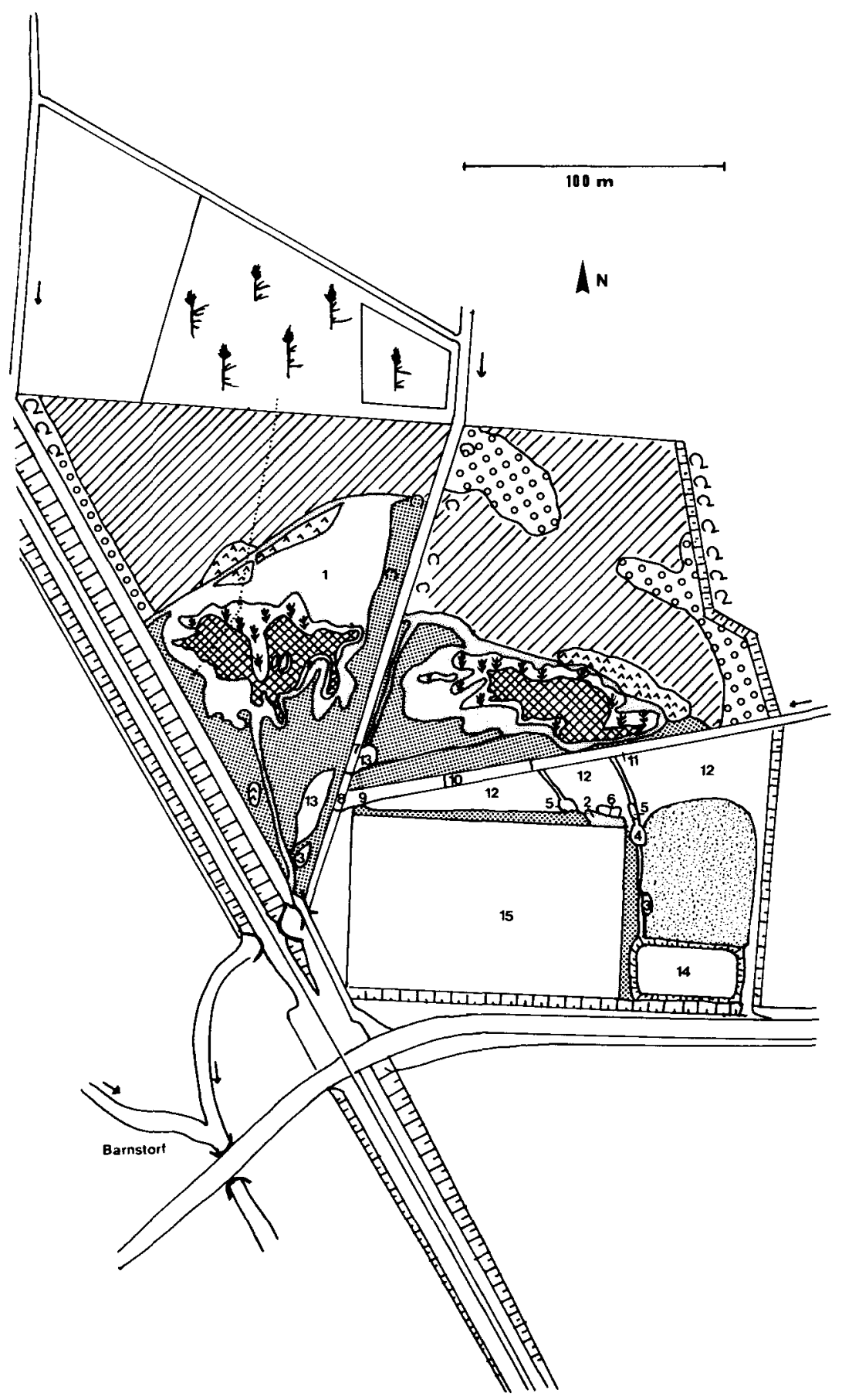

Fig. 12. Vegetationskarte der Salzwiesen bei Barnstorf, Niedersachsen (1984). 
Legende zu Fig. 12:

两

$4+4$

Vegetationsfreie Stellen.

\$ Puccinellio distantis-salicornietum ramosissimae.

Puccinellietum distantis.

1 Puccinellietum distantis mit größerem Triglochin mamitimu-Bestand.

2 Kleinflächiger Aster tripolium/Puccinellia distans-Bestand.

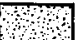

Sehr kleinräumiger wechsel zwischen vegetationsfreien stellen und Beständen von Salicomia ramosissima, Aster tripolium/Puccineliia distans, Atriplex hastata, Juncus gerardit, Phragmites australis und Agropyron repens (nicht beweidet).

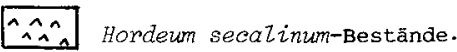

Agropyron repens-Gesellschaft.

0\%० Größere Urtica dioica-Herden oder Cirsium amense-Bestände.

Kriechrasen mit Agropyron repens, Ranunculus repens, Agrostis stoionifera, z. T. mit halotoleranten Arten (Tmifoliw fragifem, Triglochin maritimu, Juncus inflexus).

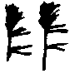

Phragmites australis

R Populus nigra-Hybrid c.

D Salix spec. c.

3 Melizotus dentata-Bestand.

4 Sonchus arvensis ssp. uliginosus (einzelne Individuen).

5 Lotus tenuis (einzelne Jndividuen)

6 Juncus gerardii-Herde.

7 Graben mit Zannichellia palustris, Böschung mit Aster tripolium, Puccinelitia distans und Atriplex hastata.

8 Nasturtium officinale-Röhricht.

9 Graben mit Bemila erecta und Mentha aquatica.

10 Graben mit Glyceria fluitans.

11 Graben mit Phragmites austratis.

12 Brache mit Urtica dioica und Heracleum sphondylium.

13 Durch Weidevieh stark zertretener Bereich mit Therophyten.

14 Anpflanzung, vor allem Posa spec, Acer campestre.

15 Sportplatz.

$\vdots \quad$ Linie des Transektes mit Höhenunterschieden (Nivellierung), vgl. Fig. 13. 


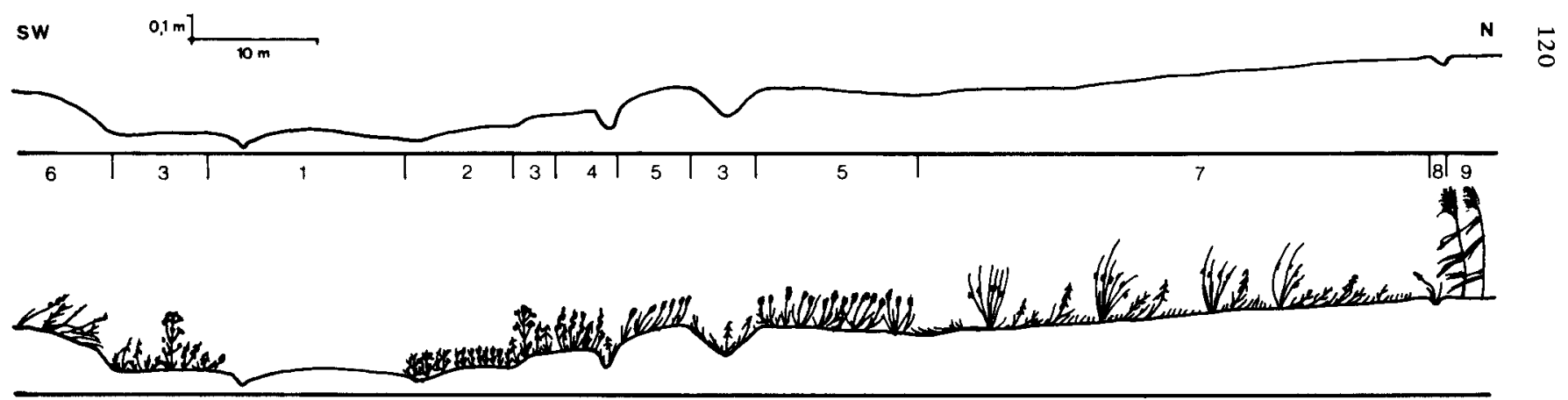

Fig. 13. Ausschnitt aus der Vegetationszonierung entlang eines Transektes (mit Höhenunterschieden), Barnstorf 1984.

Legende zu Fig. 13

1 Vegetationsfrei.

2 Puccinellio distantis-Salicomietum ramosissimae.

3 Puccinellietum distantis.

4 Triglochin maritimum-Bestand.

5 Hordeum secalinum-Bestand.

6 Agropyron repens-Gesellschaft.

7 Kriechrasen mit Juncus inflexus.

8 Graben mit Carex otrubae.

9 Pbragmites australis. 


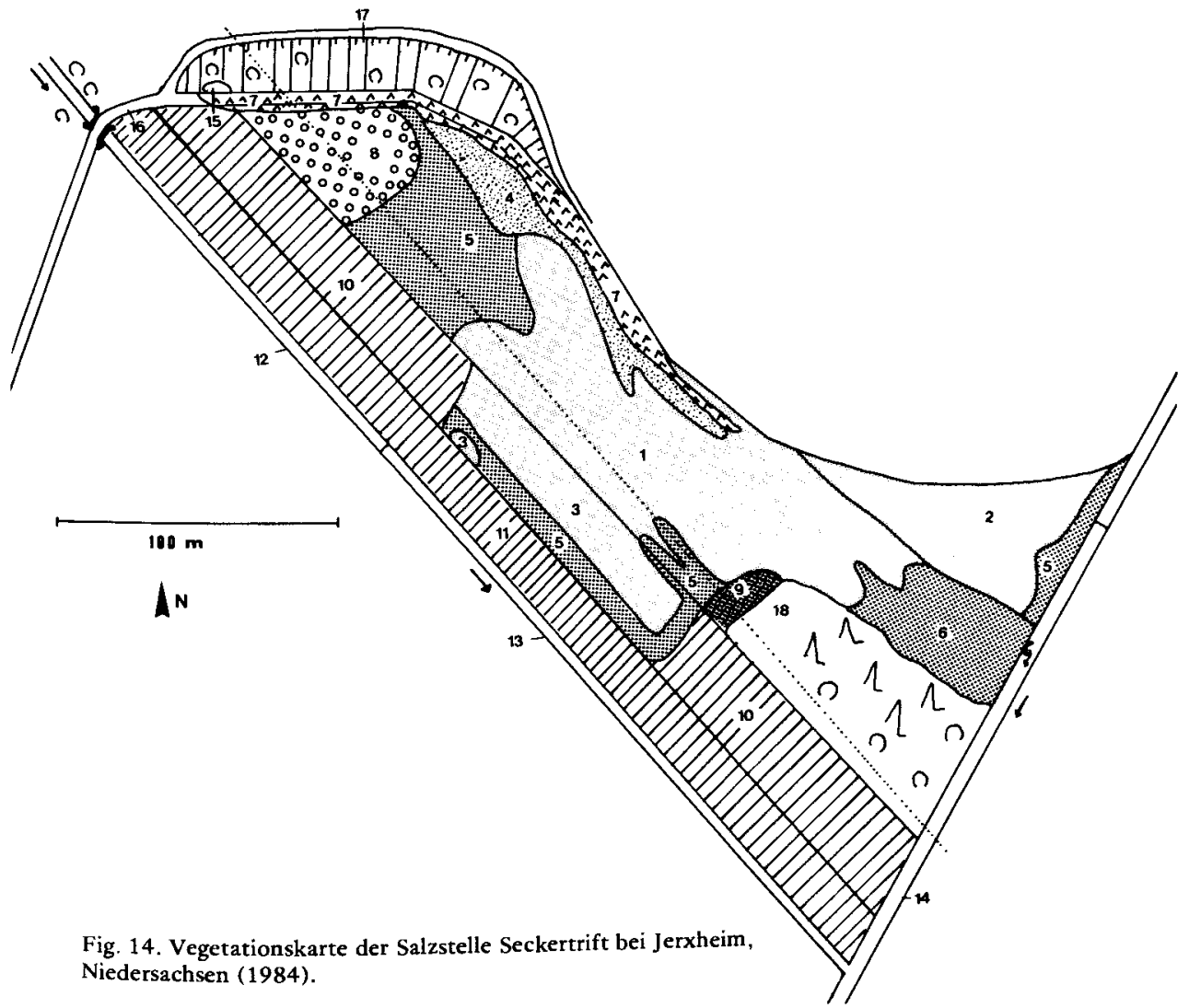

Legende zu Fig. 14:

Falophytenvegetation mit typischer zonierung (vegetationsfreie stellen, Salicornietum ramosissimae, Puccinellietum distantis und Juncetum gerardii) in mehr oder weniger kleinräumigem Wechsel.

2 Halophyten, vor allem Salicornia ramosissima, Spergularia marina, dazwischen vegetationsfreie stellen.

3 Halophytenvegetation, dazwischen grōBere Flächen mit Agropyron repens-Rasen.

04 Phragmites australis-Bestände.

Agropyron repens-Gesellschaft.

\%6 Apropyron repens- und Phragmites australis-Bestände.

An Trockenere wuchsorte mit Centaurium pulchellum und/oder Melilotus dentata. 
Legende zu Fig. 14 (Fortsetzung):

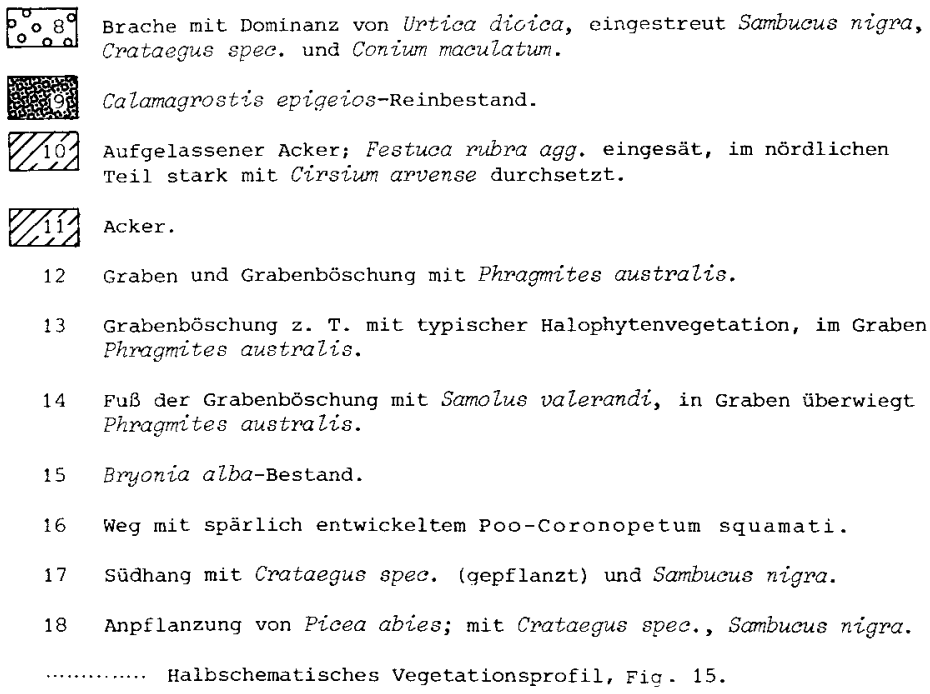

Legende zu Fig. 15:

1 Haferfeld.

2 Weg mit Agropyron repens.

3 Böschung mit Crataegus spec. und Sambucus nigra.

4 Weg mit Centaurium pulcbellum.

5 Urtica dioica-Bestand mit Sambucus nigra und Conium maculatum.

6 Agropyron repens-Geselichaft.

7 Puccinellietum distantis.

8 Puccinellio distantis-Salicornietum ramosissimae.

9 Vegetationsfrei.

10 Juncetum gerardii.

11 Tripleurospermum inodorum-Bestand.

12 Agropyron repens-Bestand.

13 Calamagrostis epigeios-Bestand.

14 Fraxinus excelsior, ca. $10 \mathrm{~m}$ hoch.

15 Anpflanzung von Picea abies (z.T. abgestorben; mit spontanem Gehölzaufkommen: Crataegus spec. und Sambucus nigra).

16 Symphoricarpos albus und Crataegus spec

17 Arrbenatberum elatius-Bestand.

18 Böschung mit Atriplex bastata und Aster tripolium.

19 Graben mit Samolus valerandi und Pbragmites australis.

20 Böschung mit Festuca rubra und Arrbenatberum elatius.

21 Weizenfeld mit Avena fatua. 
http://www.digibib.tu-bs.de/?docid=00042033

$11 / 01 / 2012$

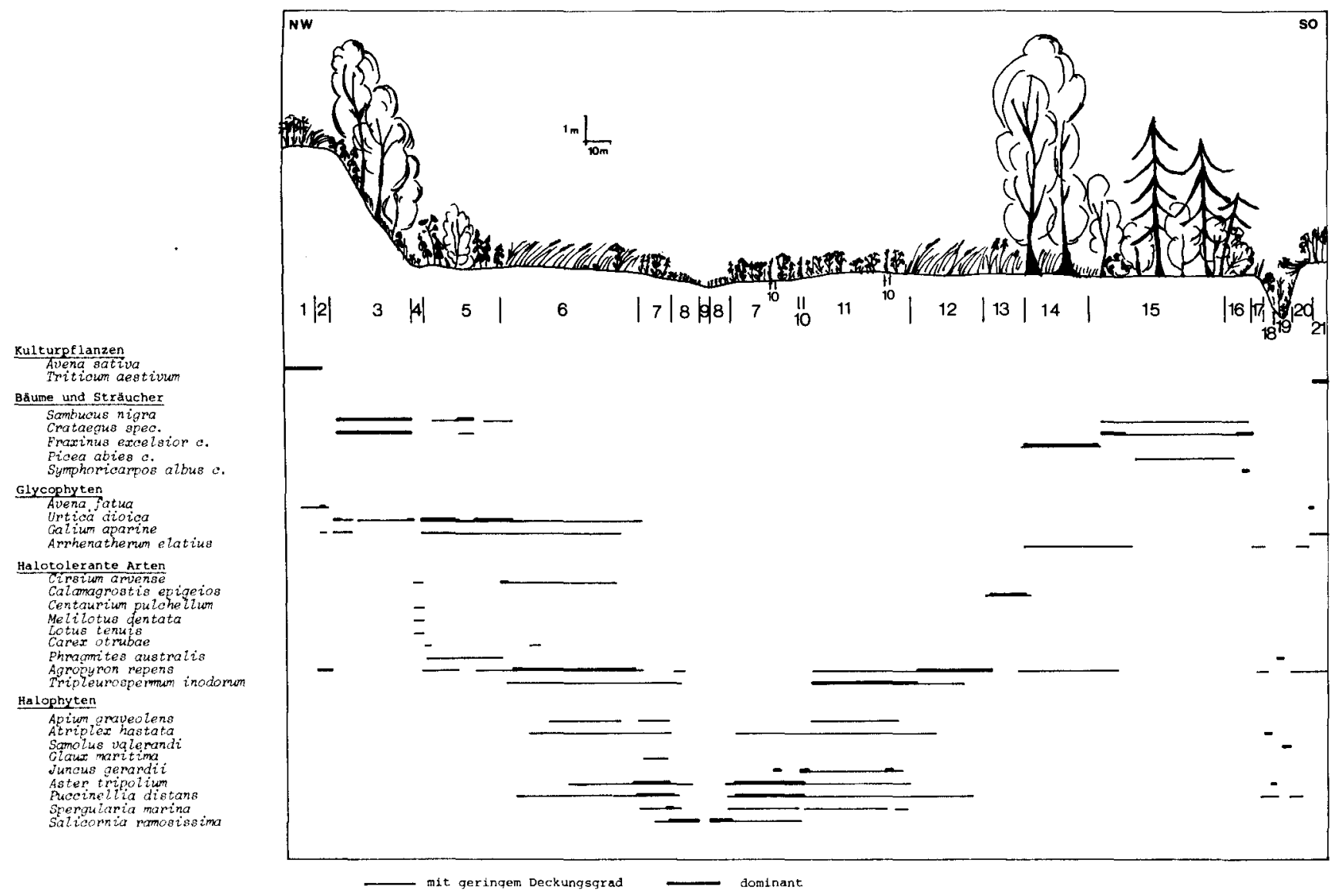

Fig. 15. Halbschematisches Vegetationsprofil durch die Seckertrift bei Jerxheim 1984, Länge ca. $400 \mathrm{~m}$. 


\section{Literatur}

Altehage, C. \& Rossmann, B. (1940): Vegetationskundliche Untersuchungen der Halophytenflora binnenländischer Salzstellen im Trockengebiet Mitteldeutschlands. - Beih. Bot. Cbl. 60 (B): 135-180, Dresden.

Balátová-Tuláčková, E. (1970): Bibliographie der Phänospektrum-Diagramme von Pflanzengesellschaften. - Excerpta Botanica, Sectio B 10 (4): 243-256, Stuttgart.

Dierschke, H. (1972): Zur Aufnahme und Darstellung phänologischer Erscheinungen in Pflanzengesellschaften. - In: Tüxen, R. (Hrsg.): Grundfragen und Methoden in der Pflanzensoziologie. - Ber. Int. Symps. Int. Vereinigg. Vegetationsk. Rinteln 1970: 291-311, Den Haag.

- (1974): Saumgesellschaften im Vegetations- und Standortgefälle an Waldrändern. Scripta Geobotanica 6: 246 S., Göttingen.

Duvigneaud, J. (1967); Flore et végétation halophiles de la Lorraine orientale (Dép. Moselle, France). - Mém. Soc. Roy. Bot. Belgique 3: 1-122, Bruxelles.

Füllekrug, E. (1967): Phänologische Diagramme aus einem Melico-Fagetum. - Mitt. flor.soz. Arb.gem. NF 11/12: 143-158, Todenmann.

- (1969): Phänologische Diagramme von Glatthaferwiesen und Halbtrockenrasen. -- Mitt. flor.-soz. Arb.gem. NF 14: 255-273, Todenmann.

Gams, H. (1918): Prinzipienfragen der Vegetationsforschung. - Vjahrsschr. Naturf. Ges. Zürich 63, Zürich.

Gilli, A. (1950): Die Phänologie des Salicornietum herbaceae an der Nordsee. - Phyton 2 (4): 247-259, Horn (Niederösterreich).

Gödde, M. (1986): Vergleichende Untersuchung der Ruderalvegetation der Großstädte Düsseldorf, Essen und Münster. - 273, S., Düsseldorf.

Janßen, Ch. (1986): Ökologische Untersuchungen an Binnensalzstellen in Südostniedersachsen. - Phytocoenologia 14 (1): 109-142, Stuttgart-Braunschweig.

Jeckel, G. (1984): Syntaxonomische Gliederung, Verbreitung und Lebensbedingungen nordwestdeutscher Sandtrockenrasen (Sedo-Scleranthetea). - Phytocoenologia 12 (1): 9153 , Stuttgart-Braunschweig.

Kratochwil, A. (1983): Zur Phänologie von Pflanzen und blütenbesuchenden Insekten (Hymenoptera, Lepidoptera, Diptera, Coleoptera) eines versaumten Halbtrockenrasens im Kaiserstuhl - ein Beitrag zur Erhaltung brachliegender Wiesen als Lizens-Biotope gefährdeter Tierarten. - Beih. Veröff. Natursch. Landschaftspfl. Bad.-Württ. 34: 57-108, Karlsruhe.

- (1984): Pflanzengesellschaften und Blütenbesucher-Gemeinschaften: biozönologische Untersuchungen in einem nicht mehr bewirtschafteten Halbtrockenrasen (Mesobrometum) im Kaiserstuhl (Südwestdeutschland). - Phytocoenologia 11 (4): 455-669, Stuttgart-Braunschweig.

Otte, A. (1986): Phänologische Beobachtungen in Hochstaudenfluren auf Kiesinseln in der Oder (SW-Harzrand). - Tuexenia 6: 105-125, Göttingen.

Schennikow, A.P. (1932): Phänologische Spektra der Pflanzengesellschaften. In: Abderhalden, E. (Ed.): Biologische Arbeitsmethoden Abt. 11, Teil 6: 251-266, Berlin.

Schmeisky, H. (1977): Einfluß von Weidetieren auf Salzpflanzengesellschaften. - Ber. Int. Symp. Int. Vereinigg. Vegetationsk. (1976): 481-498, Rinteln.

Schwabe, A. \& Kratochwil, A. (1984): Vegetationskundliche und blütenökologische Untersuchungen in Salzrasen der Nordseeinseln Borkum. - Tuexenia 4: 125-152, Göttingen.

Tüxen, R. (1962): Das phänologische Gesellschaftsdiagramm. - Mitt. flor.-soz. Arb.gem. NF 9: 51-52, Stolzenau/Weser.

Tüxen, R. \& Wojterska, M. (1977): Bibliographie der Phänospektrum-Diagramme von Pflanzengesellschaften Pars II. - Excerpta Botanica, Sectio B 16 (4): 306-317, Stuttgart.

Vogel, A. (1981): Klimabedingungen und Stickstoff-Versorgung von Wiesengesellschaften verschiedener Höhenstufen des Westharzes. - Diss. Bot. 60: 164, S., Stuttgart.

Weber, J. \& Pfadenhauer, J. (1987): Phänologische Beobachtungen auf Streuwiesen unter Berücksichtigung des Nutzungseinflusses (Rothenrainer Moorgebiet bei Bad Tölz). - Ber. Bayer. Bot. Ges. 58: 153-177, München.

Anschrift der Verfasser:

Dipl.-Biol. Christiane JANSSEN, Schunterstraße 54, D-3300 Braunschweig.

Priv--Doz. Dr. Dietmar BRANDES, Universitätsbibliothek der TU Braunschweig, Pockelstraße 13, D-3300 Braunschweig. 